\title{
NONEQUALITY OF DIMENSIONS FOR METRIC SPACES
}

BY

PRABIR ROY $\left(^{1}\right)$

Introduction. There are three classical set-theoretic notions of dimension; they are [2, p. 153]:

Small inductive dimension (= Menger-Urysohn dimension), denoted by ind such that ind $(S)=-1$ if $S$ is empty, ind $(S) \leqq n$ if for every point $p \in S$ and open set $U$ containing $p$ there is an open set $V$ satisfying

$$
p \in V \subset U, \text { ind (boundary of } V) \leqq n-1,
$$

and ind $(S)=n$ if ind $(S) \leqq n$ but ind $(S) \leqq n-1$ is not true.

Large inductive dimension (due to Urysohn), denoted by Ind such that Ind $(S)$ $=-1$ if $S$ is empty, Ind $(S) \leqq n$ if for every closed set $C \subset S$ and open set $U$ containing $C$ there is an open set $V$ satisfying

$$
C \subset V \subset U, \text { Ind (boundary of } V \text { ) } \leqq n-1,
$$

and Ind $(S)=n$ if Ind $(S) \leqq n$ but Ind $(S) \leqq n-1$ is not true.

Covering dimension (= Lebesgue covering dimension), denoted by dim such that $\operatorname{dim}(S)=-1$ if $S$ is empty, $\operatorname{dim}(S) \leqq n$ if every finite open cover of $S$ has a finite open refining cover of order $\leqq n+1$, that is, no point of $S$ belongs to more than $n+1$ members of the refinement, and $\operatorname{dim}(S)=n$ if $\operatorname{dim}(S) \leqq n$ but $\operatorname{dim}(S) \leqq n-1$ is not true.

It is well known that for separable metric space $S$

$$
\text { ind }(S)=\operatorname{Ind}(S)=\operatorname{dim}(S) \text {. }
$$

Recently Katetov showed that for any metric space $S$

$$
\text { Ind }(S)=\operatorname{dim}(S) \text {. }
$$

However, the question, is ind $(S)=\operatorname{dim}(S)$ for all metric spaces, remained open. We shall answer this question in the negative. (For a comprehensive account of the preceding material the reader is invited to consult $P$. Aleksandrov's paper [1, pp. 1-4].) We shall prove, in succeeding sections, the following statement.

THEOREM. There is a complete metric space $\Delta$ such that ind $(\Delta)=0$ but $\operatorname{dim}(\Delta)=1$.

1. Description of the space. In this section we define the points of $\Delta$ and certain subsets of $\Delta$. Although these subsets are already called regions here, the fact that

Presented to the Society, January 26, 1963 under the title Failure of equivalence of dimension concepts for metric spaces; received by the editors April 17, 1967.

(') Supported by National Science Foundation grants NSF-G21514 and NSF-GP-6951. 
they form a topological basis is proved in $\S 2$. The remarks at the end are intended to clarify some of the rather arbitrary assertions implicit in the process of defining.

1.1. Notations. The symbol $\varnothing$ as usual will denote the empty set. By a sequence we shall mean a function defined on either the set of all positive integers, or the set of all nonnegative integers, or any initial segment of either of them. With this in mind the following notations are adopted.

1.1.1. $X=$ the set of all finite sequences of real numbers, defined on initial segments of the nonnegative integers, such that if $x \in X$, then $x(i)=0$ only in case $i=0$.

1.1.2. If $x \in X$, then $|x|$ will denote the greatest integer for which $x$ is defined. ( $|x|$ may be thought of as the length of $x$.)

1.1.3. $Y=$ the set of all reversible sequences of positive numbers, defined on the set of all positive integers. The word "reversible" will be used instead of "oneto-one."

1.1.4. $Z=$ the set of all infinite sequences of positive numbers, defined on the set of all positive integers.

1.1.5. If $r$ is a positive number, then $Y_{r}$ will denote the set of all members of $Y$ which take on the value $r$, and

1.1.6. $F_{r}$ will denote a reversible function from the positive numbers onto $Y_{r}$.

1.1.7. If $K$ is a collection of sets, then $K^{*}$ will denote the union of members of $K$.

1.2. Points of $\Delta$. There are two types of points of $\Delta$ and these types will be called $P_{1}$ and $P_{2}$.

1.2.1. $P_{1}=$ the set of all sequences of nonzero real numbers, defined on the positive integers.

1.2.2. $P_{2}=X \times Y \times Z$. $\left(p_{X}, p_{Y}\right.$, and $p_{Z}$ will denote the coordinates of $p \in P_{2}$. $)$

1.3. Regions of $\Delta$. There are also two types of regions and these types will be denoted by $\Gamma_{1}$ and $\Gamma_{2}$.

$R$ is a member of $\Gamma_{1}$ only in case there is an $x \in X$ with $|x| \neq 0$ such that,

1.3.1. $R=R^{1} \cup R^{2}$, where

1.3.2. $R^{1}=\left\{p \mid p \in P_{1} ;\right.$ and $p(i)=x(i)$ for $\left.i=1, \ldots,|x|\right\}$, and

1.3.3. $R^{2}=\left\{p\left|p \in P_{2} ;\right| p_{X}|\geqq| x \mid\right.$; and $p_{X}(i)=x(i)$ for $\left.i=1, \ldots,|x|\right\}$. Such a region $R$ will be denoted by $R_{x}$.

$R$ is a member of $\Gamma_{2}$ only in case there is a point $p \in P_{2}$ and a positive integer $n$ such that,

1.3.4. $R=R^{0} \cup R^{+} \cup R^{-}$, where

1.3.5. $R^{0}=\left\{q \mid q \in P_{2} ; \quad q_{X}=p_{X} ; q_{Y}=p_{Y} ;\right.$ and if $n>1$, then $q_{Z}(i)=p_{Z}(i)$ for $i=1, \ldots, n-1\}$, and

1.3.6. $R^{+}=\bigcup_{i=1}^{\infty} R_{y\left(p, n_{1}+\right)_{i}}$ and $R^{-}=\bigcup_{i=1}^{\infty} R_{\gamma(p, n,-)}$, where $\left\{\gamma(p, n,+)_{i}\right\}_{1}^{\infty}$ and $\left\{\gamma(p, n,-)_{i}\right\}_{1}^{\infty}$ are the two infinite reversible sequences of members of $X$ such that if $j$ is a positive integer, then

1.3.7. $\left|\gamma(p, n, \pm)_{j}\right|=\left|p_{X}\right|+n+1$, 
1.3.8. $\gamma(p, n, \pm)_{j}(i)=p_{X}(i)$ for $i=0, \ldots,\left|p_{X}\right|$,

1.3.9. $\gamma(p, n, \pm)_{j}\left(\left|p_{X}\right|+1\right)= \pm p_{Y}(n+j-1)$,

1.3.10. $\gamma(p, n, \pm)_{j}\left(\left|p_{X}\right|+2\right)=\mp F_{p_{Y}(n+j-1)}^{-1}\left(p_{Y}\right)$, and if $n>1$,

1.3.11. $\gamma(p, n, \pm)_{j}\left(\left|p_{X}\right|+2+i\right)=\mp p_{Z}(i)$ for $i=1, \ldots, n-1$.

Such a region $R$ will be denoted by $R_{(p, n)}$.

1.4. Remarks. Note that 1.1.6 makes sense because $Y_{r}$ has the power of the continuum. In 1.2, $P_{1} \cap P_{2}=\varnothing$. In 1.3, the fact that $\left\{\gamma(p, n,+)_{i}\right\}_{1}^{\infty}$ and $\left\{\gamma(p, n,-)_{i}\right\}_{1}^{\infty}$ are reversible sequences follows from $1.3 .9,1.1 .3$, and 1.1 .1 , whereby $p_{Y}$ is a reversible sequence of positive numbers, and two members of $X$ are different if they differ for some integer. Also for the same reason we have that $\gamma(p, n,+)$; $\neq \gamma(p, n,-)_{k}$ for each of $j$ and $k$ an integer. Finally observe that item 1.3.10 makes sense because by 1.1.5 $p_{Y} \in Y_{p_{Y}(n+j-1)}$ and by 1.1.6, $F_{p_{Y}(n+j-1)}$ is a reversible function onto $Y_{p_{Y}(n+j-1)}$.

2. Some preliminary lemmas. Now we shall exhibit, in a series of lemmas, certain basic properties of the space $\Delta$, including a proof that the regions defined in $\S 1$, are indeed well defined. In subsequent sections these lemmas will be translated into statements asserted in the theorem.

2.1. Lemma. Suppose that each of $R_{x}$ and $R_{x^{\prime}}$ is a member of $\Gamma_{1}$ and each of $R_{(p, n)}$ and $R_{(q, m)}$ is a member of $\Gamma_{2}$.

2.1.1. $R_{x} \supset R_{x^{\prime}} \Leftrightarrow R_{x} \cap R_{x^{\prime}} \neq \varnothing$ and $|x| \leqq\left|x^{\prime}\right| \Leftrightarrow|x| \leqq\left|x^{\prime}\right|$ and $x(i)=x^{\prime}(i)$ for $i=1, \ldots,|x|$.

2.1.2. $R_{x} \supset R_{(p, n)} \Leftrightarrow R_{x} \cap R_{(p, n)}^{0} \neq \varnothing \Leftrightarrow R_{x} \supset R_{p_{x}}$.

2.1.3. $R_{(p, n)} \supset R_{x} \Leftrightarrow R_{(p, n)}^{ \pm} \supset R_{x}$.

2.1.4. $R_{(p, n)} \cap R_{(q, m)} \neq \varnothing,\left|p_{X}\right|=\left|q_{X}\right|$, and $n \leqq m \Leftrightarrow n \leqq m$ and $R_{(p, n)}^{0} \cap R_{(a, m)}^{0}$ $\neq \varnothing \Leftrightarrow R_{(p, n)} \supset R_{(q, m)}$ and $\left|p_{X}\right| \geqq\left|q_{X}\right|$.

2.1.5. $R_{(p, n)} \supset R_{(q, m)}$ and $\left|p_{X}\right|<\left|q_{X}\right| \Leftrightarrow R_{(p, n)}^{ \pm} \supset R_{(q, m)}$.

Proof. In each item we shall prove the implications pointing to the right and finally show that the last statement implies the first one.

Proof of 2.1.1. For the first implication, by 1.1.3, 1.2.2, and 1.1.1, let $p \in R_{x^{\prime}}$ with $p \in P_{2}$ and $p_{X}=x^{\prime}$. Since $p \in R_{x}$, by 1.3.3, we have $\left|x^{\prime}\right|=\left|p_{X}\right| \geqq|x|$. The next implication is immediate by applying 1.3.2 if a point $p \in P_{1}$ belongs to the intersection, and by applying 1.3.3 if a point $p \in P_{2}$ belongs to the intersection. That the first statement follows from the last one is obvious by the definition of $\Gamma_{1}$.

Proof of 2.1.2. The first implication is transparent. For the second implication let $p^{\prime}$ belong to the common part. Noting that $p_{X}^{\prime}=p_{X}$ by 1.3 .5 and $\left|p_{X}\right| \geqq|x|$ by 1.3.3, we have that $p^{\prime} \in R_{p_{X}}$ by 1.3 .3 and hence $R_{x} \supset R_{p_{X}}$ by 2.1.1. In the last implication observe that $R_{p_{X}} \supset R_{(p, n)}^{0}$ by 1.3 .5 and 1.3.3; and in view of 2.1.1, $R_{p_{X}}$ $\supset R_{(p, n)}^{ \pm}$by $1.3 .6,1.3 .7$, and 1.3.8.

Proof of 2.1.3. Assume that the first implication is false. By 1.1.1 and 1.2.2, let $p^{\prime} \in P_{2}$ with $p_{X}^{\prime}=p_{X}$ and $p_{Y}^{\prime} \neq p_{Y}$. It follows that $p^{\prime} \in R_{p_{X}}$, by 1.3 .3 ; and hence 
$p^{\prime} \in R_{x}$ by 2.1.1, in view of the assumption, but $p^{\prime} \notin R_{(p, n)}$, by $1.3 .5,1.3 .7$ and 1.3.3. The converse needs no proof.

Proof of 2.1.4. For the first implication observe that by 1.3.5, 1.3.7, and 1.3.3, we have that $R_{(p, n)}^{0} \cap R_{(q, m)}^{ \pm}=\varnothing=R_{(q, m)}^{0} \cap R_{(p, n)}^{ \pm}$. But if $R_{(p, n)}^{ \pm} \cap R_{(q, m)}^{ \pm} \neq \varnothing$, then in view of 1.3.6, 2.1.1, and 1.3.7, we have that $q_{X}=p_{X}$, by $1.3 .8, q_{Y}=p_{Y}$ by 1.3 .9 and 1.3.10, and if $n>1$, then $q_{z}(i)=p_{z}(i)$ for $i=1, \ldots, n-1$, by 1.3.11-that is, $q \in R_{(q, m)}^{0}$ $\cap R_{(p, n)}^{0}$, by 1.3.5.

For the second implication we get immediately from 1.3.5 that $q_{X}=p_{X}, q_{Y}=p_{Y}$, and if $n>1$, then $q_{z}(i)=p_{z}(i)$ for $i=1, \ldots, n-1$. Hence, recalling that $n \leqq m$, $R_{(p, n)}^{0} \supset R_{(q, m)}^{0}$ by 1.3.5. Moreover, in view of 1.3.7-1.3.11 it follows from 2.1.1 that $R_{(q, m, \pm)} \subset R_{(p, n, \pm)_{j+m-n}}$ for $j=1,2,3, \ldots$ It follows then that $R_{(p, n)} \supset R_{(q, m)}$.

Finally in the last implication we only need to prove that $\left|p_{X}\right|=\left|q_{X}\right|$ and $n \leqq m$. If on the contrary $\left|p_{X}\right|>\left|q_{X}\right|$, then by $1.3 .5,1.3 .7$, and $1.3 .3, q \notin R_{(p, n)}$. Similarly if $n>m$ then, setting, by $1.2 .2, q^{\prime} \in P_{2}$ with $q_{X}^{\prime}=q_{X}, q_{Y}^{\prime}=q_{Y}$, and if $m>1, q_{Z}^{\prime}(i)=q_{Z}(i)$ for $i=1, \ldots, m-1$, but $q_{z}^{\prime}(m) \neq p_{z}(m)$, we have that $q^{\prime} \in R_{(q, m)}^{0}$ by 1.3 .5 , but $q^{\prime}$ $\notin R_{(p, n)}$ by $1.3 .5,1.3 .7$, and 1.3.3.

Proof of 2.1.5. For the first implication note that $R_{q_{X}} \cap R_{(p, n)}^{0}=\varnothing$, for otherwise $R_{q_{X}} \supset R_{p_{X}}$ by 2.1.2, which implies by 2.1 .1 that $\left|q_{X}\right| \leqq\left|p_{X}\right|$. Hence $R_{(p, n)}^{+}$ $\cup R_{(\bar{p}, n)} \supset R_{(q, m)}$. But in view of 1.3.6, we have $R_{(p, n)}^{ \pm} \supset R_{(q, m)}$ by 2.1.2. The converse is evident in presence of 1.3.7 and 1.3.3.

2.2. Notations. Suppose that $R_{x} \in \Gamma_{1}$ and $R_{(p, n)} \in \Gamma_{2}$. The following notations are to be adopted.

2.2.1. $E_{x}=\left\{R_{(q, m)}|| q_{X}|+1=| x \mid, q_{X}(i)=x(i)\right.$ for $i=0, \ldots,\left|q_{X}\right|$, and $\left.q_{Y} \in Y_{|x(|x|)|}\right\}$.

2.2.2. $E_{(p, n)}=\bigcup_{i=1}^{\infty} E_{\gamma(p, n, \pm)_{i}}$.

(NoTE: In 2.2.1, since the only conditions on $R_{(q, m)}$ are on $q$, we have that if $R_{(q, m)}$ $\in E_{x}$ then $R_{(q, i)} \in E_{x}$ for $i=1,2,3, \ldots$ The same is obviously true for $E_{(p, n)}$.)

2.2.3. $G_{n}^{\prime}=\left\{R_{x}|| x \mid=n\right\} \cup\left\{R_{(q, m)}|| q_{x} \mid+m=n\right\}$ for $n=1,2,3, \ldots$

2.2.4. $G_{n}=\bigcup_{i=n}^{\infty} G_{i}^{\prime}$ for $n=1,2,3, \ldots$

2.3. LEMMA. If $n$ is a positive integer, then

$$
G_{n} \supset G_{n+1}, \quad\left(G_{n} \cap \Gamma_{1}\right)^{*} \supset P_{1} \text { and }\left(G_{n} \cap \Gamma_{2}\right)^{*} \supset P_{2} \text {. }
$$

Proof. The proof follows immediately from 2.2.4 and the definitions of $\Gamma_{1}$ and $\Gamma_{2}$, using members of $\Gamma_{1}$ to cover points in $P_{1}$ and using members of $\Gamma_{2}$ to cover points in $P_{2}$.

2.4. Lemma. If $R_{x} \in \Gamma_{1}$, then there is a positive integer $N$ such that if $R \in G_{N}$ and $R \notin E_{x}$ then

$$
R_{x} \supset R \Leftrightarrow R_{x} \cap R \neq \varnothing .
$$

The least such integer will be denoted by $N_{x}$.

Proof. Obviously if $R_{x} \supset R$, then $R_{x} \cap R \neq \varnothing$. But the converse needs an 
argument. For each positive integer $j$ with $1 \leqq j \leqq|x|-1$ (if such a $j$ exists), by 1.1 .5 and 1.1.6, let

$$
m_{j}=\left[F_{|x(j)|}(|x(j+1)|)\right]^{-1}(|x(j)|)
$$

Now let,

$$
\left.N=\sum_{j=1}^{|x|-1} m_{j}+|x| . \quad \text { (Note that } N=1 \text { if } x=1 .\right)
$$

Suppose that $R_{x^{\prime}} \in \Gamma_{1} \cap G_{N}$. By 2.2.3 and (2), $\left|x^{\prime}\right| \geqq N \geqq|x|$. Hence by 2.1.1, if $R_{x} \cap R_{x^{\prime}} \neq \varnothing$ then $R_{x} \supset R_{x^{\prime}}$.

Next suppose that $R_{(p, n)} \in \Gamma_{2} \cap G_{N}$ and $\left|p_{X}\right| \geqq|x|$. If $R_{x} \cap R_{(p, n)} \neq \varnothing$ then, by 2.2.2 and 2.1.1, $R_{x} \supset R_{p_{X}} \supset R_{(p, n)}$.

Consider now the case where $R_{(p, n)} \in \Gamma_{2} \cap G_{N}$ and

$$
\left|p_{X}\right|<|x| \text {. }
$$

In this situation we shall show that

$$
\text { if } R_{x} \cap R_{(p, n)} \neq \varnothing \text { then } R_{(p, n)} \in E_{x},
$$

and thus finish the proof of the lemma. Noting that $R_{x} \cap R_{(p, n)}^{0}=\varnothing$ by (3) and 2.1.2, let $x^{\prime}=\gamma(p, n, \pm)_{j}$ for some positive integer $j$ such that $R_{x} \cap R_{x^{\prime}} \neq \varnothing$. It follows that by $1.3 .7,2.2 .3$, and (2),

$$
\left|x^{\prime}\right|=\left|p_{x}\right|+n+1 \geqq N+1>|x|,
$$

and hence by (2) and (3),

$$
n>\sum_{j=1}^{|x|-1} m_{j}
$$

Hence in presence of 2.1.1 we have that

$$
x(i)=x^{\prime}(i)=P_{X}(i) \text { for } i=0, \ldots,\left|p_{X}\right|, \text { by } 1.3 .8 \text {; }
$$

$$
\left|x\left(\left|p_{X}\right|+1\right)\right|=\left|x^{\prime}\left(\left|P_{X}\right|+1\right)\right|=p_{Y}(n+j-1), \quad \text { by } 1.3 .9 \text { and 1.3.3. }
$$

We further claim that

$$
\left|p_{X}\right|+1=|x|,
$$

for otherwise: by (3), $\left|p_{X}\right|+2 \leqq|x|$, and then by 1.3.10 and (8)

$$
\left|x\left(\left|p_{X}\right|+2\right)\right|=\left|x^{\prime}\left(\left|p_{X}\right|+2\right)\right|=F_{p_{Y}(n+j-1)}^{-1}\left(p_{Y}\right)=F_{\left|x\left(\left|p_{X}\right|+1\right)\right|}^{-1}\left(p_{Y}\right),
$$

which implies in view of (1) that

$$
\begin{aligned}
F_{\left|x\left(\left|p_{X}\right|+1\right)\right|}\left(\left|x\left(\left|p_{X}\right|+2\right)\right|\right)(n+j-1) & =p_{Y}(n+j-1)=\left|x\left(\left|p_{X}\right|+1\right)\right| \\
& =F_{\left|x\left(\left|p_{X}\right|+1\right)\right|}\left(\left|x\left(\left|p_{X}\right|+2\right)\right|\right)\left(m_{\left|p_{X}\right|+1}\right),
\end{aligned}
$$


and hence by $1.1 .5,1.1 .6$, and 1.1.3, we have that

$$
n+j-1=m_{\left|p_{x}\right|+1}
$$

namely, a contradiction to (6). Now, in view of (9), (8) implies that $p_{Y} \in Y_{|x(|x|)|}$, and this together with (9) and (7) are exactly the conditions (see 2.2.1) that $R_{(p, n)}$ $\in E_{x}$. Thus (4) is established and the proof of the lemma is completed.

2.5. LemMA. If $R_{x} \in \Gamma_{1}$ and $R_{(p, n)} \in E_{x}$ then

$$
R_{x} \cap R_{(p, n)}=\varnothing \Leftrightarrow n \geqq p_{Y}^{-1}(|x(|x|)|)+1 .
$$

Proof. Suppose that $j$ is a positive integer so that $n+j=p_{\bar{Y}}^{-1}(|x(|x|)|)+1$. Now in view of 2.2.1 we have that: $|x|=\left|p_{X}\right|+1<\left|p_{X}\right|+n+1=\left|\gamma(p, n, \pm)_{j}\right|$, by 1.3.7; $x(i)=p_{X}(i)=\gamma(p, n, \pm)_{j}(i)$ for $i=1, \ldots,\left|p_{X}\right|$, by 1.3 .8 ; and $x(|x|)= \pm p_{Y}(n+j-1)$ $=\gamma(p, n, \pm)_{j}(|x|)$, by 1.3.9. Therefore, by 2.1 .1 , if $x(|x|)<0$ then $R_{x} \supset R_{y(p, n,-)}$ but if $x(|x|)>0$ then $R_{x} \supset R_{y(p, n,+)}$-and hence $R_{x} \cap R_{(p, n)} \neq \varnothing$.

Conversely suppose that $n \geqq p_{Y}^{-1}(x(|x|))+1$. Recalling that $\left|p_{X}\right|+1=|x|$, we get that $R_{x} \cap R_{(p, n)}^{0} \neq \varnothing$ by 1.3 .5 and 1.3.3. Also if $j$ is a positive integer then: $\left|\gamma(p, n, \pm)_{j}\right|>\left|p_{X}\right|+1=|x|$, by 1.3 .7 , but $\gamma(p, n, \pm)_{j}(|x|)= \pm p_{Y}(n+j-1) \neq x(|x|)$, by 1.3.9 due to 1.1 .3 and the fact that $n+j-1>n-1 \geqq p_{Y}^{-1}(|x(|x|)|)$-and hence $R_{x} \cap R_{r(p, n, \pm) ;} \neq \varnothing$ by 2.1.1. Thus $R_{x} \cap R_{(p, n)}=\varnothing$.

2.6. LemMA. If $R_{(p, n)} \in \Gamma_{2}$, then there exists a positive integer $N$ such that if $R \in G_{N}$ and $R \notin E_{(p, n)}$ then

$$
R_{(p, n)} \supset R \Leftrightarrow R_{(p, n)} \cap R \neq \varnothing .
$$

The least such positive integer $N$ will be denoted by $N_{(p, n)}$.

Proof. The implication to the right is transparent. But the converse requires an explanation. First let

$$
N=\left|p_{X}\right|+n+1 \text {. }
$$

Suppose that $R_{x} \in \Gamma_{1} \cap G_{N}$. Since $|x| \geqq N>\left|p_{X}\right|$ by 2.2 .4 and (1), we have that $R_{x} \cap R_{(p, n)}^{0}=\varnothing$ by 1.3 .5 and 1.3.3. Hence if $R_{x} \cap R_{(p, n)} \neq \varnothing$ then, by 1.3 .7 and 2.1.1, $R_{(\mathrm{p}, n)}^{ \pm} \supset R_{x}$.

In case $R_{(q, m)} \in \Gamma_{2} \cap G_{N}$ and $\left|q_{X}\right|>\left|p_{X}\right|+n$, we get by 1.3 .5 and 1.3 .3 that $R_{a_{X}} \cap R_{(p, n)}^{0}=\varnothing$. Hence if $R_{(q, m)} \cap R_{(p, n)} \neq \varnothing$, then by 2.1.2, 1.3.7, and 2.1.1, $R_{(p, n)}^{ \pm} \supset R_{q_{X}}$-that is $R_{(p, n)} \supset R_{(q, m)}$.

Consider now the case where $R_{(q, m)} \in \Gamma_{2} \cap G_{N}$ and

$$
\left|q_{x}\right| \leqq\left|p_{X}\right|+n \text {. }
$$

First note that by 2.2 .4

$$
\left|q_{X}\right|+m \geqq\left|p_{X}\right|+n .
$$


Observe that in case $\left|p_{X}\right|=\left|q_{X}\right|$, then $n \leqq m$ by (3)-hence if $R_{(p, n)} \cap R_{(q, m)} \neq \varnothing$ then $R_{(p, n)} \supset R_{(q, m)}$ by 2.1.4.

On the other hand we shall show that

$$
\text { if } R_{(p, n)} \cap R_{(q, m)} \neq \varnothing \quad \text { and }\left|p_{X}\right| \neq\left|q_{X}\right| \text { then } R_{(q, m)} \in E_{(p, n)},
$$

and thereby complete the proof of the lemma. Noting that $R_{(p, n)}^{0} \cap R_{(q, m)}^{0}=\varnothing$ by 2.1.4 due to (2), and $R_{(p, n)}^{0} \cap R_{(q, m)}^{ \pm}=\varnothing=R_{(q, m)}^{0} \cap R_{(p, n)}^{ \pm}$by 1.3.5, 1.3.7, and 1.3.3 due to (2) and (3), we let $\dot{x}=\gamma(p, n, \pm)_{j}$ for some $j$ and $x^{\prime}=\gamma(q, m, \pm)_{k}$ for some $k$ so that $R_{x} \cap R_{x^{\prime}} \neq \varnothing$. But then in view of 2.1.1 we get:

$$
\left|x^{\prime}\right|=\left|q_{X}\right|+m+1 \geqq\left|p_{X}\right|+n+1=|x| \geqq\left|q_{X}\right|+1,
$$

by $1.3 .7,(3)$ and (2),

$$
q_{x}(i)=x^{\prime}(i)=x(i) \text { for } i=0, \ldots,\left|q_{X}\right|,
$$

by 1.3 .8 and (5), and

$$
q_{Y}(m+k-1)=\left|x^{\prime}\left(\left|q_{X}\right|+1\right)\right|=\left|x\left(\left|q_{X}\right|+1\right)\right|
$$

by 1.3 .9 and (5). Furthermore we claim that

$$
\left|q_{X}\right|=\left|p_{X}\right|+n,
$$

for otherwise, recalling that $\left|p_{X}\right|=\left|q_{X}\right|$ is ruled out by assumption, we have that if $\left|q_{X}\right|<\left|p_{X}\right|$ then by $1.3 .11, x^{\prime}\left(\left|p_{X}\right|+1\right) \cdot x^{\prime}\left(\left|p_{X}\right|+2\right)>0$, which implies that $x\left(\left|p_{X}\right|+1\right)$ $\cdot x\left(\left|p_{X}\right|+2\right)>0$ a contradiction to 1.3 .9 and 1.3.10, and similarly if $\left|p_{X}\right|+n$ $>\left|q_{X}\right|>\left|p_{X}\right|$, then $x\left(\left|q_{X}\right|+1\right) x\left(\left|q_{X}\right|+2\right)>0$, whereby $x^{\prime}\left(\left|q_{X}\right|+1\right) \cdot x^{\prime}\left(\left|q_{X}\right|+2\right)>0$ again a contradiction to 1.3.9 and 1.3.10. Now in view of (8), (5) says that $\left(\left|q_{x}\right|+1\right)$ $=|x|$ and (7) says that $q_{Y} \in Y_{|x(|x|)|}$, and these together with (6) are exactly the conditions (see 2.2.1) that $R_{(q, m)} \in E_{x}$, which means that, recalling $x=\gamma(p, n, \pm)_{j}$, $R_{(q, m)} \in E_{(p, n)}$ by 2.2.2. Thus (4) is established, whereby the proof of the lemma is completed.

2.7. LEMMA. The regions are well defined, that is, if $U$ and $V$ are regions with a point $p^{\prime} \in U \cap V$, then there is a region $W$ such that $p \in W \subset U \cap V$.

Proof. Case 1. Suppose that $U=R_{x} \in \Gamma_{1}$ and $V=R_{x^{\prime}} \in \Gamma_{1}$ with $|x| \leqq\left|x^{\prime}\right|$, we have immediately from 2.1 .1 that $p^{\prime} \in R_{x^{\prime}}=R_{x} \cap R_{x^{\prime}}$.

Case 2. Suppose now that $U=R_{x} \in \Gamma_{1}$ but $V=R_{(q, m)} \in \Gamma_{2}$. If $R_{x} \cap R_{(a, m)}^{0} \neq \varnothing$, then, by 2.1.2, $p^{\prime} \in R_{(q, m)}=R_{x} \cap R_{(q, m)}$. If $R_{x} \cap R_{(q, m)}^{0}=\varnothing$, then

$$
p^{\prime} \in R_{x} \cap R_{(q, m)}=R_{x} \cap\left[R_{(q, m)}^{+} \cup R_{(q, m)}^{-}\right]
$$

but since $R_{(q, m)}^{ \pm}$are unions of members of $\Gamma_{1}$, the problem is reduced to Case 1 .

Case 3. Consider now the case where $U=R_{(p, n)} \in \Gamma_{2}$ and $V=R_{(q, m)} \in \Gamma_{2}$ with $n \leqq m$. If $R_{(p, n)}^{0} \cap R_{(q, m)}^{0} \neq \varnothing$ then, by $2.1 .4, p^{\prime} \in R_{(q, m)}=R_{(p, n)} \cap R_{(q, m)}$. If $R_{(p, n)}^{0}$ $\cap R_{(q, m)}^{0}=\varnothing$ then

$$
p^{\prime} \in R_{(p, n)} \cap R_{(q, m)}=\left[R_{(p, n)} \cap\left(R_{(q, m)}^{+} \cup R_{(q, m)}^{-}\right)\right] \cup\left[R_{(q, m)} \cap\left(R_{(p, n)}^{+} \cup R_{(p, n)}^{-}\right)\right]
$$


but since $R_{(p, n)}^{ \pm}$and $R_{(q, m)}^{ \pm}$are unions of members of $\Gamma_{1}$, the problem is reduced to Case 2.

2.8. LeMMA. Suppose that $U$ is a region containing a point $p$.

2.8.1. If $p \in P_{1}$ then there is a region $R_{x} \in \Gamma_{1}$ with $p \in R_{x} \subset U$.

2.8.2. If $p \in P_{2}$, there is a positive integer $n$ with $p \in R_{(p, n)} \subset U$.

Proof of 2.8.1. We only need to consider the case where $U=R_{(q, m)} \in \Gamma_{2}$. By Lemma 2.3, let $p \in R_{x} \in G_{N_{(q, m)}}$ (see Lemma 2.6 for the definition of $N_{(q, m)}$ ). Since $R_{x} \notin E_{(q, m)}$, we have by Lemma 2.6 that $p \in R_{x} \subset R_{(q, m)}=U$.

Proof of 2.8.2. If $U=R_{x} \in \Gamma_{1}$, then $\left|p_{X}\right| \geqq|x|$ by 1.3.3. Hence $R_{\left(p, N_{x}\right)} \notin E_{x}$, by 2.2.1. (See Lemma 2.5 for the definition of $N_{x}$.) Now applying Lemma 2.4 we have that $p \in R_{\left(p, N_{x}\right)} \subset U$.

If $U=R_{(q, m)} \in \Gamma_{2}$, then by $1.3 .5,1.3 .7$, and 1.3 .3 either $\left|p_{X}\right|=\left|q_{X}\right|$ or $\left|p_{X}\right| \geqq\left|q_{X}\right|$ $+m+1$. Consequently by 2.2 .2 and $2.2 .1, R_{\left(p, N_{(q, m)}\right.} \notin E_{(q, m)}$. Now applying Lemma 2.6, $p \in R_{\left(p, N_{(q, m)}\right.} \subset U$.

3. Metrization of the space $\Delta$. Here we show that the space $\Delta$ is metrizable, that is, there is a metric on $\Delta$ which is compatible with the topology of $\Delta$. This is done by employing a metrization theorem due to $R$. L. Moore. (It is unfortunate that this beautiful and eminently useful theorem (stated as Lemma 3.3), proved by R. L. Moore in [4], is not yet widely known.)

\subsection{Lemma. The space $\Delta$ is a Hausdorff space.}

Proof. Suppose that $p$ and $q$ are two points in $P_{1}$. By 1.2 .1 , let $j$ be a positive integer for which $p(j) \neq q(j)$. Now by 1.1 .1 , let $x$ and $x^{\prime}$ belong to $X$ so that: $|x|=\left|x^{\prime}\right|=j$, and $x(i)=p(i), x^{\prime}(i)=q(i)$ for $i=1, \ldots, j$. It follows that $p \in R_{x}$ and $q \in R_{x^{\prime}}$ by 1.3 .2 , but $R_{x} \cap R_{x^{\prime}}=\varnothing$ by 2.1.1.

Now suppose that $p \in P_{1}$ and $q \in P_{2}$. By 1.1.1, let $x \in X$ such that: $|x|=\left|q_{x}\right|+2$, and $x(i)=p(i)$ for $i=1, \ldots,|x|$. We have $q \in R_{\left(q, N_{x}\right)}$ by 1.3.5 and $p \in R_{x}$ by 1.3.2. But noting that $R_{\left(q, N_{x}\right)} \notin R_{x}$ by 2.1 .2 and 2.1.1, and $R_{\left(q, N_{x}\right)} \notin E_{x}$ by 2.2 .1 , we conclude, by Lemma 2.4 , that $R_{\left(a, N_{x}\right)} \cap R_{x}=\varnothing$.

Finally suppose that $p \in P_{2}$ and $q \in P_{2}$ with $\left|p_{X}\right| \geqq\left|q_{X}\right|$. In case $p_{X}=q_{X}$ and $p_{Y}=q_{Y}$, then by fixing, in view of 1.2.2 and 1.1.4, $j$ to be a positive integer with $p_{z}(j) \neq q_{z}(j)$, we get by 1.3 .5 that $p \in R_{(p, j+1)}$ and $q \in R_{(q, j+1)}$, but $R_{(p, j+1)}^{0} \cap R_{(q, j+1)}^{0}=\varnothing$, which implies by 2.1.4 that $R_{(p, j+1)} \cap R_{(q, j+1)}=\varnothing$. On the other hand suppose that it is not true that $p_{X}=q_{X}$ and $p_{Y}=q_{Y}$. By 1.3.5, $p \in R_{(p, 1)}$ and $q \in R_{\left(q, N_{(p, 1)}\right)}$. But since $\left|p_{x}\right| \geqq\left|q_{x}\right|$, we get that $R_{\left(q, N_{(p, 1)}\right.} \notin R_{(p, 1)}$ by 2.1 .4 and 1.3.5, and $R_{\left(q, N_{(p, 1)}\right)} \notin E_{(p, 1)}$ by 2.2.2 and 2.2.1. Now applying Lemma $2.5, R_{(p, 1)} \cap R_{\left(q, N_{(p, 1)}\right)}=\varnothing$.

3.2. LEMMA. If $U$ is a region containing a point $p$ then there is a region $V$ and $a$ positive integer $N$ such that $p \in V \subset U$, and if $W \in G_{N}$ and $W \cap V \neq \varnothing$ then $W \subset U$. 
Proof. Suppose that $p \in P_{1}$. By Lemma 2.8 and 1.3.2, let $R_{x} \in \Gamma_{1}$ such that $x(i)=p(i)$ for $i=1, \ldots,|x|$ and $p \in R_{x} \subset U$. Clearly it is sufficient to prove the lemma for $R_{x}$ instead of $U$. By 1.1.1, let $x^{\prime} \in X$ such that: $\left|x^{\prime}\right|=|x|+1 ; x^{\prime}(i)=x(i)$ for $i=1, \ldots,|x|$; and $x^{\prime}\left(\left|x^{\prime}\right|\right)=p(|x|+1)$. It is evident that $p \in R_{x^{\prime}} \subset R_{x}$, by 1.3.2 and 2.1.1. Now let $N=N_{x^{\prime}}$. In case $W \in G_{N}$ and $W \notin E_{x^{\prime}}$, it follows from Lemma 2.4 that if $W \cap R_{x^{\prime}} \neq \varnothing$ then $W \subset R_{x^{\prime}} \subset R_{x}$. In case $W \in G_{N}$ and $W=R_{(q, m)} \in E_{x^{\prime}}$ then noting that, by 2.2.1 and 1.3.3, $q \in R_{x}$, we have, by 2.1.2, $W=R_{(q, m)} \subset R_{x}$.

Suppose now that $p \in P_{2}$. By Lemma 2.8 and 1.3.5, let $n$ be a positive integer with $p \in R_{(p, n)} \subset U$. As before it suffices to prove the lemma for $R_{(p, n)}$ instead of $U$. First note that by 2.1.4, $p \in R_{(p, n+1)} \subset R_{(p, n)}$. Now let $N=N_{(p, n+1)}$. In case $W \in G_{N}$ and $W \notin E_{(p, n+1)}$, then it follows from Lemma 2.6 that: if $W \cap R_{(p, n+1)} \neq \varnothing$ then $W \subset R_{(p, n+1)}$. Now assume that $W \in G_{N}$ and $W=R_{(q, m)} \in E_{(p, n+1)}$. In this situation we shall show that

$$
W=R_{(q, m)} \subset R_{(p, n)}^{ \pm} .
$$

By 2.2.2 and without loss of generality, let $j$ be a positive integer with $R_{(q, m)} \in$ $E_{\gamma(p, n+1,+),}$. Now in view of 2.2.1, an inspection of 1.3.7-1.3.11 will show that $R_{(q, m)} \subset R_{y(p, n,+)_{1+1}}$. Thus (1) is verified, whereby the proof of Lemma 3.2 is completed.

3.3. Lemma (MoOre's Metrization Theorem [4]). A topological space $S$ is metrizable if

(1) the space $S$ is Hausdorff, and

(2) there is a decreasing sequence $G_{1} \supset G_{2} \supset G_{3} \supset \ldots$ of open coverings of $S$ such that if $U$ is an open set containing a point $p$, then there is a positive integer $N$ with the property that: if $R_{1}, R_{2} \in G_{N}, p \in R_{1}$, and $R_{1} \cap R_{2} \neq \varnothing$, then $R_{1} \cup R_{2} \subset U$.

3.4. Property I. The space $\Delta$ is metrizable.

Proof. We shall apply Lemma 3.3. First Lemma 3.1 says that $\Delta$ is Hausdorff, and Lemma 2.3 gives us that $G_{1}, G_{2}, G_{3}, \ldots$, as defined in 2.2 .4 , is a decreasing sequence of open coverings of $\Delta$.

Now suppose that $U$ is a region containing a point $p$. By Lemma 3.2 let $N_{1}$ be a positive integer and $V_{1}$ be a region such that $p \in V_{1} \subset U$ and if $W \in G_{N_{1}}$ and $W \cap V_{1} \neq \varnothing$ then $W \subset U$.

Applying Lemma 3.2 again, this time to $V_{1}$, let $N_{2}$ and $V_{2}$ be appropriate objects. Now set $N=N_{1}+N_{2}$.

If $R_{1}, R_{2} \in G_{N}, p \in R_{1}$ and $R_{1} \cap R_{2} \neq \varnothing$, then $R_{1} \in G_{N_{2}}$ and $R_{1} \cap V_{2} \neq \varnothing$. Hence $R_{1} \subset V_{1}$. Therefore $R_{2} \cap V_{1} \neq \varnothing$ and $G_{2} \in G_{N_{1}}$. Hence $R_{2} \subset U$. Thus, $R_{1} \cup R_{2} \subset U$.

4. Completeness of the space $\Delta$. Since completeness may be considered as a natural extension of the notion of compactness, and as the various dimensions are the same for compact metric spaces [1, p. 2], it is somewhat curious that the space $\Delta$ turns out to be complete. In any case the completeness of $\Delta$ will be used to compute the dimensions of $\Delta$ in the later sections. 
4.1. Property II. The space $\Delta$ is complete, that is, if $H_{1}, H_{2}, H_{3}, \ldots$ is an infinite sequence of regions and $C_{1}, C_{2}, C_{3}, \ldots$ is an infinite sequence of closed sets such that for each positive integer $i$

$$
\varnothing \neq C_{i+1} \subset C_{i} \subset H_{i} \in G_{i},
$$

then $\bigcap_{i=1}^{\infty} C_{i} \neq \varnothing$.

Proof. First note that if $\left\{C_{i}^{\prime}\right\}_{1}^{\infty}$ is an infinite subsequence of $\left\{C_{i}\right\}_{1}^{\infty}$, then $\bigcap_{1}^{\infty} C_{i}$ $=\bigcap_{1}^{\infty} C_{i}^{\prime}$.

In case for infinitely many positive integers $i, H_{i} \in \Gamma_{1}$, by applying 2.2.3 in view of 2.2.4, we select a subsequence $\left\{R_{x_{i}}\right\}_{1}^{\infty}$ of $\left\{H_{i}\right\}_{1}^{\infty}$ such that for each positive integer $i,\left|x_{i}\right|<\left|x_{i+1}\right|$ and $R_{x_{i}} \in G_{i}$. Let $\left\{C_{i}^{\prime}\right\}_{1}^{\infty}$ be the corresponding subsequence of $\left\{C_{i}\right\}_{1}^{\infty}$. Observe that if $i$ is a positive integer, then $C_{i+1}^{\prime} \subset R_{x_{i}} \cap R_{x_{i+1}} \neq \varnothing$-hence by 2.1.1, $R_{x_{i}} \supset R_{x_{i+1}}$ and $x_{i}(j)=x_{i+1}(j)$ for $j=1, \ldots,\left|x_{i}\right|$. Now we can let $p \in P_{1}$ with: $p(j)=x_{1}(j)$ for $1 \leqq j \leqq\left|x_{1}\right|$, and $p(j)=x_{i+1}(j)$ for $\left|x_{i}\right| \leqq j \leqq\left|x_{i+1}\right|$. It follows by Lemma 2.8 and 2.1 .1 that $p \in \bigcap_{i=1}^{\infty} C_{i}^{\prime}$. Consequently $\bigcap_{i=1}^{\infty} C_{i} \neq \varnothing$.

In case there is an integer $M$ with $H_{i}=R_{\left(q_{i}, m_{i}\right)} \in \Gamma_{2}$ for $i>M$, and $\left\{\left|\left(q_{M+i}\right)_{X}\right|\right\}_{1}^{\infty}$ is an unbounded sequence of positive integers, by 2.2 .3 in view of 2.2 .4 , we choose a subsequence $\left\{\boldsymbol{R}_{\left(p_{i}, n_{i}\right)}\right\}_{1}^{\infty}$ of $\left\{H_{i}\right\}_{1}^{\infty}$ and a corresponding subsequence $\left\{\boldsymbol{C}_{i}^{\prime}\right\}_{1}^{\infty}$ of $\left\{\boldsymbol{C}_{i}\right\}_{1}^{\infty}$, such that $\left|\left(p_{i}\right)_{X}\right|>i$ for each positive integer $i$. Consequently, for each positive integer $i, C_{i}^{\prime} \subset R_{\left(p_{i}, n_{i}\right)} \subset R_{\left(p_{i}\right)_{X}} \in G_{i}$, by 2.1 .2 and 2.2.4. Thus the problem is reduced to the previous case, whereby $\bigcap_{1}^{\infty} C_{i}=\bigcap_{1}^{\infty} C_{i}^{\prime} \neq \varnothing$.

Finally suppose that as above there is a positive integer $M$ with $H_{i}=R_{\left(q_{i}, m_{i}\right)} \in \Gamma_{2}$ for $i>M$, but now $\left\{\left|\left(q_{M+i}\right)_{X}\right|\right\}_{1}^{\infty}$ is a bounded sequence-some integral value being taken on infinitely many times. Hence by 2.2 .3 and 2.2 .4 we select a subsequence $\left\{R_{\left(p_{i}, n_{i}\right)}\right\}_{1}^{\infty}$ of $\left\{H_{i}\right\}_{1}^{\infty}$ and the corresponding subsequence $\left\{C_{i}^{\prime}\right\}_{1}^{\infty}$ of $\left\{C_{i}\right\}_{1}^{\infty}$ such that for each positive integer $i,\left|\left(p_{i}\right)_{X}\right|=\left|\left(p_{i+1}\right)_{X}\right|, n_{i}<n_{i+1}$, and $R_{\left(p_{i}, n_{i}\right)} \in G_{i}$. It follows that if $i$ is a positive integer, then $C_{i+1} \subset R_{\left(p_{i}, n_{i}\right)} \cap R_{\left(p_{i+1}, n_{i+1}\right)} \neq \varnothing$, and hence by 2.1.4 $R_{\left(p_{i}, n_{i}\right)} \supset R_{\left(p_{i+1}, n_{i+1}\right)}$ and $R_{\left(p_{i}, n_{i}\right)}^{0} \cap R_{\left(p_{i+1}, n_{i+1}\right)}^{0} \neq \varnothing$. Now we can let $p \in P_{2}$ such that: $p_{X}=\left(p_{1}\right)_{X}, p_{Y}=\left(p_{1}\right)_{Y}, p_{Z}(j)=\left(p_{2}\right)_{Z}(j)$ for $1 \leqq j \leqq n_{2}-1$, and $p_{Z}(j)=$ $\left(p_{i+2}\right)_{z}(j)$ for $n_{i+1}-1 \leqq j \leqq n_{i+2}-1$ for $i=1,2,3, \ldots$ It is evident by Lemma 2.8 and 2.1.4 that $p \in \bigcap_{1}^{\infty} C_{i}^{\prime}$. Consequently, $\bigcap_{1}^{\infty} C_{i} \neq \varnothing$. Thus the completeness of $\Delta$ is established.

5. Dimensions of the space $\Delta$. In this section we shall show that the ind $(\Delta)=0$ but $\operatorname{dim}(\Delta)=1$. The latter will be proved by first showing that Ind $(\Delta)>0$, then using Katetov's result that Ind $(\Delta)=\operatorname{dim}(\Delta)$ for all metric spaces, and finally demonstrating that $\operatorname{dim}(\Delta) \leqq 1$.

5.1. LemMA. Each region of $\Delta$ is closed.

Proof. Suppose that $R_{x} \in \Gamma_{1}$ and $p \in \Delta-R_{x}$. In case $p \in P_{1}$ or $p \in P_{2}$ but $R_{(p, 1)}$ $\notin E_{x}$, by Lemmas 2.3 and 2.8 let $R \in G_{N_{x}}$ so that $p \in R \notin E_{x}$. It follows by Lemma 2.4 that $R \cap R_{x}=\varnothing$. In case $p \in P_{2}$ and $R_{(p, 1)} \in E_{x}$, then putting $n=p_{Y}^{-1}(|x(|x|)|)$ 
+1 , we have by Lemma 2.5 that $p \in R_{(p, n)}$ but $R_{(p, n)} \cap R_{x}=\varnothing$. Hence each region in $\Gamma_{1}$ is a closed set.

Suppose now that $R_{(p, n)} \in \Gamma_{2}$ and $q \in \Delta-R_{(p, n)}$. In case $q \in P_{1}$ or $q \in P_{2}$ but $R_{(q, 1)} \notin E_{(p, n)}$, by Lemmas 2.3 and 2.8 , let $R \in G_{N_{(p, n)}}$ such that $q \in R \notin E_{(p, n)}$. It follows by Lemma 2.6 that $R \cap R_{(p, n)}=\varnothing$. In case $q \in P_{2}$ and $R_{(q, 1)} \in E_{(p, n)}$, by 2.2.2 and 2.2.1 let $x=\gamma(p, n, \pm)_{k}$ for some positive integer $k$ such that $R_{(q, 1)} \in E_{x}$. Now put $m=p_{Y}^{-1}(|x(|x|)|)+1$. Noting that by 2.2.1 and 1.3.7 $\left|q_{X}\right|=\left|p_{X}\right|+n$ we have that: $R_{(q, m)} \cap R_{x}=\varnothing$ by Lemma $2.5, R_{q_{X}} \cap R_{(p, n)}^{0}=\varnothing$ by 1.3 .5 and 1.3.3, and for $j \neq k, R_{q_{X}} \cap R_{y(p, n, \pm),}=\varnothing$, by 2.1 .1 because $q_{X}\left(\left|p_{X}\right|+1\right)=\gamma(p, n, \pm)_{k}\left(\left|p_{X}\right|+1\right)$ $=p_{Y}(n+k-1) \neq p_{Y}(n+j-1)=\gamma(p, n, \pm)_{j}\left(\left|p_{X}\right|+1\right)$, in view of 2.2.1, 1.3.9, 1.1.3, and 1.3.9 respectively. Consequently, $R_{(q, m)} \cap R_{(p, n)}=\varnothing$, but $q \in R_{(q, m)}$. Hence each region in $\Gamma_{2}$ is a closed set.

5.2. Property III. The small inductive dimension of $\Delta$ is zero.

Proof. If $U$ is an open set containing a point $p$ then by Lemma 2.7 there is a region $R$ with $p \in R \subset U$, and by Lemma 5.1

$$
\text { ind (boundary of } R)=\text { ind }(\varnothing)=-1 \text {. }
$$

5.3. Notations and Definitions. We shall adopt the following terms.

5.3.1. $\Pi=$ the set of all finite sequences of positive numbers, defined on initial segments of the set of positive integers. If $\pi \in \Pi$, then $|\pi|=$ the greatest integer for which $\pi$ is defined.

5.3.2. $K$ is an indicator means that $K$ is a subset of $\Pi$ with

(1) if $\pi, \pi^{\prime} \in K$, then $|\pi|=\left|\pi^{\prime}\right|$, and that integer is denoted by $|K|$,

(2) $\{r \mid r=\pi(1)$ for some $\pi \in K\}$ is an infinite set, and

(3) if $\pi \in K$ and $j$ is a positive integer with $j<|\pi|$, then $\left\{r \mid r=\pi^{\prime}(j+1)\right.$ for some $\pi^{\prime} \in K$ with $\pi^{\prime}(i)=\pi(i)$ for $\left.i=1, \ldots, j\right\}$ is an infinite set.

5.3.3. If $K$ is an indicator and $x \in X$ then

$$
\begin{aligned}
\sum(K, x \pm)=\left\{R_{x^{\prime}}|| x^{\prime} \mid\right. & =|x|+|K|, x^{\prime}(i)=x(i) \text { for } i=0, \ldots,|x|, \\
& \text { and for some } \left.\pi \in K, x^{\prime}(|x|+i)= \pm \pi(i) \text { for } i=1, \ldots,|K|\right\} .
\end{aligned}
$$

5.3.4. If $K$ is an indicator and $R_{(p, n)} \in \Gamma_{2}$ then

$$
\begin{aligned}
\sum(K,(p, n))=\left\{R_{(q, m)} \mid\right. & q \in R_{(p, n)}^{0}, m=n+|K|, \\
& \text { and for some } \left.\pi \in K, q_{z}(n-1+i)=\pi(i) \text { for } i=1, \ldots,|K|\right\} .
\end{aligned}
$$

5.4. LemMA. If $H$ is a collection of regions and $R_{(p, n)} \in \Gamma_{2}$ such that, for each $q \in R_{(p, n)}^{0}$, there is an indicator $K$ with $\sum(K,(q, n+1)) \subset H$, then there is an indicator $K^{\prime}$ such that $\sum\left(K^{\prime},(p, n)\right) \subset H$.

Proof. For each positive number $r$, let $q_{r} \in R_{(p, n)}^{0}$ with $\left(q_{r}\right)_{z}(n)=r$, and let $K_{r}$ be an indicator with $\sum\left(K_{r},\left(q_{r}, n+1\right)\right) \subset H$. Since $\left\{M|M=| K_{r} \mid\right.$ for some $\left.r\right\}$ is a countable set of integers, let $N$ be a positive integer and $T$ be an infinite set of positive numbers such that if $r \in T$ then $\left|K_{r}\right|=N$. Define $K^{\prime}$ by requiring that 
$\pi^{\prime} \in K^{\prime}$ only in case: $\left|\pi^{\prime}\right|=N+1, \pi^{\prime}(1) \in T$, and for some $\pi \in K_{\pi^{\prime}(1)}, \pi^{\prime}(i+1)=\pi(i)$ for $i=1, \ldots, N$. Clearly $K^{\prime}$ is an indicator. It follows that

$$
\sum\left(K^{\prime},(p, n)\right)=\bigcup_{r \in T} \sum\left(K_{r},\left(q_{r}, n+1\right)\right) \subset H .
$$

5.5. LEMMA. If $H$ is a collection of regions and $R_{(p, 1)} \in \Gamma_{2}$ has the property that for each $q \in R_{(p, 1)}^{0}$ there is a positive integer $M$ with $R_{(q, m)} \in H$ for $m \geqq M$, then there is an indicator $K$ such that $\sum(K,(p, 1)) \subset H$.

Proof. Suppose on the contrary that there exists no such $K$. By Lemma 5.4, let $Q$ be a sequence such that $Q_{1}=R_{(p, 1)}$, and if $Q_{n}=R_{(q, n)}$ then $Q_{n+1}=R_{\left(Q^{\prime}, n+1\right)}$ where $q^{\prime} \in R_{(q, n)}^{0}$ and there is no indicator $K$ such that $\sum\left(K,\left(q^{\prime}, n+1\right)\right) \subset H$. It is evident that by 2.1.4, Lemma 5.1, and 2.2.4, $\left\{Q_{n}\right\}_{1}^{\infty}$ is a decreasing sequence of open and closed sets with $Q_{n} \in G_{n}$ for each positive integer $n$. Therefore due to the completeness of $\Delta, \bigcap_{1}^{\infty} Q_{n} \neq \varnothing$, and in particular in view of the last paragraph of 5.1, there is a point $p^{\prime} \in R_{(p, 1)}^{0}$ with $p^{\prime} \in \bigcap_{1}^{\infty} Q_{n}$. It follows, in presence of 2.1.4, that there is no integer $M$ such that $R_{\left(p^{\prime}, M\right)} \in H$-a contradiction to the hypothesis.

5.6. LEMMA. If $R_{(p, 1)} \in \Gamma_{2}$ and $U_{1}$ and $U_{2}$ are open sets with $R_{(p, 1)}^{0} \subset U_{1} \cup U_{2}$, then there is an indicator $K$ and a positive integer $j$ with $j=1$ or $j=2$ such that $[\Sigma(K,(p, 1))]^{*} \subset U_{j}$.

Proof. We shall construct such a $K$. By Lemmas 2.7 and 2.8 let: $H_{1}=\left\{R_{(q, m)} \mid q\right.$ $\in R_{(p, 1)}^{0}$ and $\left.R_{(q, m)} \subset U_{1}\right\}, H_{2}=\left\{R_{(q, m)} \mid q \in R_{(p, 1)}^{0}\right.$ and $\left.R_{(q, m)} \subset U_{2}\right\}$, and $H=H_{1} \cup H_{2}$. By Lemma 5.5 let $K^{\prime}$ be an indicator such that $\sum\left(K^{\prime},(p, 1)\right) \subset H$. For each $k^{\prime} \in K^{\prime}$ let $\dot{q}_{k^{\prime}} \in R_{(p, 1)}^{0}$ such that $\left(q_{k^{\prime}}\right)_{Z}(i)=k^{\prime}(i)$ for $i=1, \ldots,\left|K^{\prime}\right|$. Now define two sequences $\left\{J_{i}^{1}\right\}_{1}^{\left|X^{\prime}\right|}$ and $\left\{J_{i}^{2}\right\}_{1}^{\left|K^{\prime}\right|}$ by requiring that: $\pi \in J_{1}^{1}$ (or $J_{1}^{2}$ ) only in case

$$
\pi \in K^{\prime} \quad \text { and } \quad R_{\left(q_{\pi},\left|K^{\prime}\right|+1\right)} \in H_{1} \quad\left(\text { or } H_{2}\right) \text {, }
$$

and inductively if $1 \leqq n<\left|K^{\prime}\right|$ then $\pi \in J_{n+1}^{1}$ (or $J_{n+1}^{2}$ ) only in case

$$
|\pi|=\left|K^{\prime}\right|-n+1
$$

and

(3) $\quad \pi(i)=\pi^{\prime}(i)$ where $i=1, \ldots,|\pi|$, for infinitely many $\pi^{\prime} \in J_{n}^{1}$ (or $J_{n}^{2}$ ).

By (1) it is clear that $J_{1}^{1} \cup J_{1}^{2}=K^{\prime}$. Furthermore by an inductive argument one can show that if $1 \leqq n<\left|K^{\prime}\right|$ then $J_{n+1}^{1} \cup J_{n+1}^{2}=\left\{\pi|| \pi|=| K^{\prime} \mid-n+1\right.$; and $\pi(i)=\pi^{\prime}(i)$ where $i=1, \ldots,|\pi|$, for some $\left.\pi^{\prime} \in K^{\prime}\right\}$. Hence by part (2) of 5.3.2 let $j$ be a positive integer such that $J_{\left|K^{\prime}\right|}^{j}$ is an infinite set. Now define $K$ by requiring that $\pi \in K$ only in case for each $1 \leqq n \leqq\left|K^{\prime}\right|$,

$$
\text { there is a } \pi^{\prime} \in J_{\left|K^{\prime}\right|-n+1}^{j} \text { with } \pi(i)=\pi^{\prime}(i) \text { for } i=1, \ldots, n \text {. }
$$

By the definition of $\left\{J_{i}^{j}\right\}_{i=1}^{\infty}$ and in particular (3), we have that $K$ is an indicator. Moreover by (1) it is evident that

$$
\sum(K,(p, 1)) \subset\left\{R_{\left(q_{\pi},\left|K^{\prime}\right|+1\right)} \mid \pi \in J_{1}^{j}\right\} \subset H_{j} .
$$

Consequently, $[\Sigma(K,(p, 1))]^{*} \subset U_{j}$. 
5.7. Lemma. If $K$ is an indicator, the point $p \in P_{2}$, and $x=\gamma(p, 1, \pm)_{j}$ with $j \geqq|K|+1$, then

$$
\left(\sum(K, x \mp)\right)^{*} \subset\left(\sum(K,(p, 1))\right)^{*} .
$$

Proof. For each $\pi \in K$, let $x_{\pi \mp} \in X$ such that

$$
\begin{aligned}
\left|x_{\pi \mp}\right| & =|x|+|K|, \quad x_{\pi \mp}(i)=x(i) & & \text { for } i=0, \ldots,|x|, \\
x_{\pi \mp}(|x|+i) & =\mp \pi(i) & & \text { for } i=1, \ldots,|\pi|,
\end{aligned}
$$

and let $q_{\pi} \in P_{2}$ such that $q_{\pi} \in R_{(p, 1)}^{0},\left(q_{\pi}\right)_{2}(i)=\pi(i)$ for $i=1, \ldots,|\pi|$.

We have then by 5.3.3 and 5.3.4, that if $\pi \in K$ then

$$
R_{x_{n} \mp} \in \sum(K, x \mp) \text { and } R_{\left(q_{\pi}, 1+|K|\right)} \in \sum(K,(p, 1)) ;
$$

furthermore, in presence of the definitions of $x_{\pi \mp}$ and $q_{\pi}$, an inspection of 1.3.71.3.11 will show that,

$$
x_{\pi \mp}=\gamma\left(q_{\pi}, 1+|K|, \pm\right)_{j-|K|} .
$$

Now since by 5.3.3 and 5.3.4,

and

$$
\sum(K, x \mp)=\left\{R_{x_{\pi} \mp} \mid \pi \in K\right\}
$$

we have that

$$
\sum(K,(p, 1))=\left\{R_{\left(q_{\pi}, 1+|K|\right)} \mid \pi \in K\right\},
$$

$$
\left(\sum(K, x \mp)\right)^{*} \subset\left\{R_{\left(q_{n}, 1+|K|\right)}^{\mp} \mid \pi \in K\right\}^{*} \subset\left(\sum(K,(p, 1))\right)^{*} .
$$

5.8. Lemma. Suppose that $x \in X, U_{1}$ and $U_{2}$ are open sets with $U_{1} \cup U_{2}=\Delta$, and each of $K_{1}$ and $K_{2}$ is an indicator. If $\left(\sum\left(K_{1}, x+\right)\right)^{*} \subset U_{1}$ and $\left(\sum\left(K_{2}, x-\right)\right)^{*} \subset U_{2}$ then there exist: $x^{\prime} \in X$ with $\left|x^{\prime}\right|=|x|+1$ and $x^{\prime}(i)=x(i)$ for $i=0, \ldots,|x|$, and indicators $K_{3}$ and $K_{4}$ such that

$$
\left(\sum\left(K_{3}, x^{\prime}+\right)\right)^{*} \subset U_{1} \text { and }\left(\sum\left(K_{4}, x-\right)\right)^{*} \subset U_{2} .
$$

Proof. The proof will consist of exhibiting $x^{\prime}, K_{3}$, and $K_{4}$. Let $\sigma_{1}$ and $\sigma_{2}$ be reversible sequences into

(1) $\left\{r \mid r=k_{1}(1)\right.$ for some $\left.k_{1} \in K_{1}\right\}$ and $\left\{r \mid r=k_{2}(1)\right.$ for some $\left.k_{2} \in K_{2}\right\}$

respectively with disjoint ranges. Let $\sigma$ be a sequence such that for each positive integer $n$,

$$
\sigma(2 n-1)=\sigma_{1}(n) \text { and } \sigma(2 n)=\sigma_{2}(n)
$$

Clearly $\sigma \in Y$. Let

$$
S=\{y \mid y \in Y \text { and } \sigma \text { is a subsequence of } y\},
$$

and for each $s \in S$ let $q_{s}$ be a point such that

$$
q_{s} \in P_{2}, \quad\left(q_{s}\right)_{X}=x, \quad\left(q_{s}\right)_{Y}=s .
$$


By application of Lemma 5.6 to $R_{\left(q_{s}, 1\right)}$ for each $s \in S$ and noting that $S$ is uncountable, we obtain without loss of generality a set $S^{\prime}$ such that

$$
S^{\prime} \subset S, \quad S^{\prime} \text { is uncountable, }
$$

$s^{\prime} \in S^{\prime}$ implies there is a $K_{s^{\prime}}$ with $\left(\sum\left(K_{s^{\prime}},\left(q_{s^{\prime}}, 1\right)\right)^{*} \subset U_{1}\right.$.

But $\left\{|K| \mid K=K_{s^{\prime}}\right.$ for some $\left.s^{\prime} \in S\right\}$ is a countable set of integers. Consequently let $N$ be a positive integer and $T$ be a set such that

$$
T \subset S^{\prime}, \quad T \text { is infinite, } t \in T \text { implies that }\left|K_{t}\right|=N .
$$

Define $x^{\prime} \in X$ as follows:

$$
\left|x^{\prime}\right|=|x|+1, \quad x^{\prime}\left(\left|x^{\prime}\right|\right)=-\sigma_{2}(N), \quad x^{\prime}(i)=x(i) \quad(i=0, \ldots,|x|) .
$$

Define $K_{3}$ by requiring that $\pi \in K_{3}$ only in case:

$$
\begin{aligned}
|\pi| & =N+1, \pi(1)=F_{\sigma_{2}(N)}^{-1}(t) & & \text { (for some } t \in T) \\
\pi(i+1) & =\pi^{\prime}(i) \quad \text { where } i=1, \ldots, N & & \text { for some } \pi^{\prime} \in K_{t} .
\end{aligned}
$$

By (6), (1), and 1.1.6 it is evident that $K_{3}$ is an indicator. Define $K_{4}$ by requiring that $\pi \in K_{4}$ only in case $|\pi|=\left|K_{2}\right|$, if $\left|K_{2}\right|>1$ then $\pi(i)=\pi^{\prime}(i+1)\left(i=1, \ldots,\left|K_{2}\right|-1\right)$, for some $\pi^{\prime} \in K_{2}$ with $\pi^{\prime}(1)=\sigma_{2}(N)$.

In view of the definition of $\sigma_{2}$ it is clear that $K_{4}$ is an indicator. Moreover by (1) and (7) we have that

$$
\left(\sum\left(K_{4}, x^{\prime}\right)\right)^{*} \subset\left\{R_{x^{\prime \prime}} \mid R_{x^{*}} \in \sum\left(K_{2}, x-\right) \text { and } x^{\prime \prime}(|x|+1)=-\sigma_{2}(N)\right\}^{*},
$$

and hence from the hypothesis

$$
\left(\sum\left(K_{4}, x^{\prime}-\right)\right)^{*} \subset\left(\sum\left(K_{2}, x-\right)\right)^{*} \subset U_{2} .
$$

For the remaining assertion note that by (8) and 5.3.3,

$$
\sum\left(K_{3}, x^{\prime}+\right)=\bigcup_{t \in T} \sum\left(K_{t}, x_{t}+\right),
$$

where for $t \in T$,

$$
\begin{aligned}
\left|x_{t}\right| & =|x|+2 ; x_{t}(|x|+1)=-\sigma_{2}(N) ; x_{t}(|x|+2)=F_{\sigma_{2}(N)}^{-1}(t) \\
x_{t}(i) & =x(i) \text { for } i=0, \ldots,|x| .
\end{aligned}
$$

But now observe that for each $t \in T$ we have that by (4) and 1.3.7-1.3.11,

$$
x_{t}=\gamma\left(q_{t}, 1,-\right)_{m_{t}},
$$

where $m_{t}=t^{-1}\left(\sigma_{2}(N)\right) \geqq N=\left|K_{t}\right|$, by (2) and (6), and hence applying Lemma 5.7,

$$
\left(\sum\left(K_{t}, x_{t}+\right)\right)^{*} \subset\left(\sum\left(K_{t},\left(q_{t}, 1\right)\right)\right)^{*} .
$$


It now follows in view of (9) and by (6) and (5) that

$$
\left(\sum\left(K_{3}, x^{\prime}+\right)\right)^{*} \subset \bigcup_{t \in T}\left(\sum\left(K_{t},\left(q_{t}, 1\right)\right)\right)^{*} \subset U_{1} .
$$

5.9. LemMA. The large inductive dimension of $\Delta$ is greater than zero.

Proof. We shall exhibit two mutually exclusive closed sets which are not separated by $\varnothing$. Let $x \in X$ with $|x|=0, K_{1}$ and $K_{2}$ be indicators with $\left|K_{1}\right|=\left|K_{2}\right|=2$, and let

$$
C_{1}=\text { closure of }\left(\sum\left(K_{1}, x+\right)\right)^{*}, \quad C_{2}=\text { closure of }\left(\sum\left(K_{2}, x-\right)\right)^{*} .
$$

It is clear that

$$
\begin{aligned}
& \text { if } p \in C_{1} \cap P_{1} \text { then } p(1)>0, \\
& \text { if } p \in C_{2} \cap P_{1} \text { then } p(1)<0, \\
& \text { if } p \in C_{1} \cap P_{2} \text { then } p_{X}(1)>0, \\
& \text { if } p \in C_{2} \cap P_{2} \text { then } p_{X}(1)<0 .
\end{aligned}
$$

Consequently, $C_{1} \cap C_{2}=\varnothing$. Suppose now that $U_{1}$ and $U_{2}$ are open sets with

$$
\Delta=U_{1} \cup U_{2}, \quad C_{1} \subset U_{1}, \quad C_{2} \subset U_{2} .
$$

In view of (1) and (2) and by repeated application of Lemma 5.8, we get a decreasing sequence $\left\{R_{x_{i}}\right\}_{1}^{\infty}$ of open and closed sets such that if $i$ is a positive integer,

$$
R_{x_{i}+1} \subset R_{x_{i}} \in G_{i}, \quad R_{x_{i}} \cap U_{1} \neq \varnothing, \quad R_{x_{i}} \cap U_{2} \neq \varnothing .
$$

Since the space $\Delta$ is complete, let $p \in \bigcap_{i=1}^{\infty} R_{x_{i}}$. By Lemma 2.8 and (3) we get that $p$ is a limit point of both $U_{1}$ and $U_{2}$. Consequently by (2), $p \in U_{1} \cap U_{2} \neq \varnothing$. Therefore $C_{1}$ and $C_{2}$ cannot be separated by $\varnothing$.

5.10. Lemma (Katetov [1, p. 3], [3]). If $S$ is a metric space then Ind $(S)=$ $\operatorname{dim}(S)$.

5.11. Property IV. The covering dimension of $\Delta$ is greater than zero.

The proof consists of Lemma 5.9 followed by Lemma 5.10 .

5.12. Property V. The covering dimension of $\Delta$ is less than or equal to 1 .

Proof. The proof rests on the following three observations:

(1) for each positive integer $n, G_{n}^{\prime}$ is an open cover with order $\leqq 2$,

(2) $G_{n+1}^{\prime}$ is a refinement of $G_{n}^{\prime}$, and

(3) each member of $G_{n}^{\prime}$ is a union of members of $G_{n+1}^{\prime}$.

The proofs of these observations are contained in the arguments given for Lemmas 2.3-2.6. Now let $K$ be a finite open cover of $\Delta$. Let $H_{1}$ be the collection to which $U$ belongs if and only if $U \in G_{1}^{\prime}$ and $U$ is a subset of some member of $K$. For each positive integer $n>1$, let $H_{n}$ be a collection to which $U$ belongs if and only if $U \in G_{n}^{\prime}, U$ is a subset of some member of $K$, but $U$ is not a subset of $\bigcup_{i=1}^{n-1} H_{i}^{*}$. It is clear that $\bigcup_{i=1}^{\infty} H_{i}$ is an open cover refining $K$. Moreover, $\bigcup_{i=1}^{\infty} H_{i}$ is of order $\leqq 2$, for othe rwise, let $p \in \Delta$ be a point belonging to more than two members of $\bigcup_{i=1}^{\infty} H_{i}$ 
say $U_{1}, U_{2}$ and $U_{3}$ labelled in such a manner that if $U_{i} \in H_{k_{i}}, i=1,2,3$, then $k_{1} \leqq k_{2} \leqq k_{3}$. Now let $V_{1}$ and $V_{3}$ be members of $G_{k_{2}}^{\prime}$ such that $p \in V_{1} \subset U_{1}$ and $p \in V_{3} \supset U_{3}$. By definition of $H_{i}$ 's, $V_{1}, U_{2}$, and $V_{3}$ are three distinct members of $G_{k_{2}}^{\prime}$ each containing $p$. This contradicts the fact that $G_{k_{2}}^{\prime}$ is of order $\leqq 2$. Now label the members of $K$ as $K=\left\{J_{1}, J_{2}, \ldots, J_{n}\right\}$, and for each $1 \leqq j \leqq n$, let $L_{j}$ be the collection to which $U$ belongs if and only if $U \in\left(\bigcup_{i=1}^{\infty} H_{i}\right) \cap J_{j}$ but $U$ is not a subset of $J_{i}$ for any $i<j$. We have that $L_{i} \cap L_{j}=\varnothing$ for $i \neq j$ and that $\bigcup_{i=1}^{n} L_{i}=\bigcup_{i=1}^{\infty} H_{i}$. So, $\left\{L_{i}^{*}\right\}_{i=1}^{n}$ is a finite open cover of $\Delta$ refining $K$ and is of order $\leqq 2$.

5.13. PROPERTY VI. The covering dimension of $\Delta$ is 1 .

6. Concluding remarks. For some conditions under which the dimensions of a metric space agree, the reader is referred to [5]. There he will also find references to other works on such conditions. The author takes this opportunity to thank Louis F. McAuley for introducing the author to the problem discussed here and encouraging him to study it.

\section{BIBLIOGRAPHY}

1. P. S. Aleksandrov, The present status of the theory of dimension, Uspehi Mat. Nauk 6 (1951), no. 5 (45), 43-68; Amer. Math. Soc. Transl. (2) 1 (1955), 1-26.

2. W. Hurewicz and H. Wallman, Dimension theory, Princeton Math. Series No. 4, Princeton Univ. Press, Princeton, N. J., 1948.

3. M. Katekov, On the dimension of metric spaces, Dokl. Akad. Nauk SSSR 79 (1951), 189-191. (Russian)

4. R. L. Moore, A set of axioms for plane analysis situs, Fund. Math. 25 (1935), 13-28.

5. L. F. McAuley, Conditions for the equality of the inductive dimensions, Portugal. Math. 24 (1965), 21-30.

The Institute for Advanced Study, Princeton, New Jersey

RUtGers, The State University, New Brunswick, New Jersey 